\title{
Iterative operator splitting method for capillary formation model in tumor angiogenesis problem: Analysis and application
}

\author{
Nurcan Gücüyenen*,† and Gamze Tanoğlu \\ Izmir Institute of Technology, Department of Mathematics, Gulbahce Campus, Urla, Izmir 35430, Turkey
}

\begin{abstract}
SUMMARY
Iterative operator splitting method is used to solve numerically the mathematical model for capillary formation in tumor angiogenesis problem. The method is based on first splitting the complex problem into simpler sub-problems. Then each sub-equation is combined with iterative schemes. The algorithms are obtained by applying the proposed method to the given model problem. The explicit local error bounds are derived to show consistency. We also explained the stability by constructing the stability functions. The obtained numerical results show that iterative splitting method provides high accuracy and efficiency with respect to other classical methods in the literature. Copyright (C) 2011 John Wiley \& Sons, Ltd.
\end{abstract}

Received 22 September 2010; Revised 4 January 2011; Accepted 23 January 2011

KEY WORDS: iterative operator splitting method; capillary formation; tumor angiogenesis; stability and consistency analysis

\section{INTRODUCTION}

The iterative splitting is a recent popular technique which is based on first splitting the complex problem into simpler differential equations. Then each sub-equation is combined with the iterative schemes, each of which is efficiently solved with suitable integrators, see [1-6]. In this paper, we deal with the iterative operator splitting scheme for the numerical solution of the nonlinear capillary formation model in tumor angiogenesis problem.

The biological model problem, see [7-9], is well suited for iterative operator splitting method since the model can be reduced to advection-diffusion-reaction problem after making a suitable transformation. This model problem has been solved by the method of lines in [10] and the tau method in [11], but they deal with more complicated systems. Since the proposed method is based on the decomposition idea, it is more attractive and more easy to apply for such models.

We also deal with the convergency analysis of the proposed method. In [1], the consistency and stability of this method are studied based on the matrix representation. In this paper, the local error bounds are derived for each iteration. The stability function is also obtained in the same manner to explain stability. Our analysis shows that the iterative splitting method provides uniformly convergent solution for the model problem.

The paper is organized as follows. Our mathematical model, capillary formation in the tumor angiogenesis problem, is introduced in Section 2. In the next section, the algorithm of the iterative splitting method is described. The application of our method to the mathematical model is given in Section 4. The error bounds are derived in Section 5. In Section 6, we deal with the stability

\footnotetext{
${ }^{*}$ Correspondence to: Nurcan Gücüyenen, Izmir Institute of Technology, Department of Mathematics, Gulbahce Campus, Urla, Izmir 35430, Turkey.

${ }^{\dagger}$ E-mail: nurcangucuyenen@iyte.edu.tr 
analysis for proposed method based on A-stability by constructing stability functions [3]. The numerical results are presented in Section 7. Finally, we discuss our results in conclusion part.

\section{MATHEMATICAL MODEL}

The mathematical model for capillary formation in tumor angiogenesis is originally presented in [7]. In this model, Levine et al. [7] used the cell transport (chemotactic) equations and developed the model by using the theory of reinforced random walk derived by David [8] and this model was recently used by Othmer and Stevens [9] to model fruiting bodies.

In this model, Levine et al. [7] introduces the following initial boundary value problem and this problem describes the endothelial cell movement in capillary.

$$
\frac{\partial u}{\partial t}=D \frac{\partial}{\partial x}\left(u \frac{\partial}{\partial x}\left(\ln \frac{u}{f(x)}\right)\right), \quad x \in(0,1), \quad t \in(0, T],
$$

where $T$ is the total time. Initial condition is given by

$$
u(x, 0)=1, \quad x \in(0,1),
$$

and boundary conditions are given by

$$
\begin{gathered}
\left.D u \frac{\partial}{\partial x}\left(\ln \frac{u}{f(x)}\right)\right|_{(0, t)}=0, \quad t \in[0, T], \\
\left.D u \frac{\partial}{\partial x}\left(\ln \frac{u}{f(x)}\right)\right|_{(1, t)}=0, \quad t \in[0, T],
\end{gathered}
$$

where $f(x)$ is the so-called transition probability function that has the effect of biasing the random walk of endothelial cells and is given by

$$
f(x)=\left(\frac{a+A_{1} x^{k}(1-x)^{k}}{b+A_{1} x^{k}(1-x)^{k}}\right)^{\alpha_{1}}\left(\frac{c+1-A_{2} x^{k}(1-x)^{k}}{d+1-A_{2} x^{k}(1-x)^{k}}\right)^{\alpha_{2}} .
$$

In this initial boundary value problem (1)-(4), $u(x, t)$ is the concentration of Endothelial Cells, $D$ is the cell diffusion constant and $a, b, c, d, A_{1}, A_{2}, \mathrm{k}, \alpha_{1}, \alpha_{2}$ are some arbitrary constants, see $[10,11]$.

\section{ITERATIVE OPERATOR SPLITTING METHOD}

Consider the abstract Cauchy problem

$$
\begin{aligned}
& u^{\prime}(t)=(A+B) u(t), \quad t \in[0, T], \\
& u(0)=u_{0},
\end{aligned}
$$

where $A$ and $B$ are linear operators and $u_{0}$ is the initial condition.

The method is based on iteration by fixing the splitting discretization step size $\tau$ on time interval $\left[t^{n}, t^{n+1}\right]$. The following algorithms are then solved consecutively for $i=1,3, \ldots, 2 p+1$.

$$
\begin{gathered}
u_{i}^{\prime}(t)=A u_{i}(t)+B u_{i-1}(t) \quad \text { with } u_{i}\left(t^{n}\right)=u^{n}, \\
u_{i+1}^{\prime}(t)=A u_{i}(t)+B u_{i+1}(t) \quad \text { with } u_{i+1}\left(t^{n}\right)=u^{n},
\end{gathered}
$$

where $u^{n}$ is the known split approximation at the time-level $t=t^{n}$ and $u_{0} \equiv 0$ is our initial guess. The split approximation at the time-level $t=t^{n+1}$ is defined as $u^{n+1}=u_{2 p+2}\left(t^{n}\right)$, see $[1,4]$. 
The iterative operator splitting can be proposed as an effective solver method for large systems of partial differential equations. This is because the local splitting error of the method can be reduced by using more iterations. All operators are used together in each iteration, resulting in better presentation of the physical problem. Larger time-steps are possible in each iterative step due to separate equations. In addition, CPU time can also be reduced by solving the splitted problem instead of full problem. The details can be found in [1,2,4-6].

\section{APPLICATION OF ITERATIVE SPLITTING METHOD TO MATHEMATICAL MODEL}

In this section, first we make a transformation to the mathematical model and have a simple equation. Second, we split this equation into two parts, then apply suitable difference approximation techniques for each derivatives and have linear bounded systems. Finally, we combine these systems with iterative schemes and apply the midpoint rule.

Considering Equation (1), it can be written as

$$
D \frac{\partial}{\partial x}\left(u \frac{\partial}{\partial x}\left(\ln \frac{u}{f(x)}\right)\right)=D \frac{\partial}{\partial x}\left(u\left(\frac{u^{\prime}}{u}-\frac{f^{\prime}(x)}{f(x)}\right)\right)
$$

and by setting $F(x)=f^{\prime}(x) / f(x)$, we have the simplified form

$$
u_{t}=D\left(u_{x x}-(u F(x))_{x}\right)
$$

The initial condition is

$$
u(x, 0)=1, \quad x \in(0,1),
$$

and boundary conditions (3), (4) become

$$
\begin{aligned}
& \left.D\left(\frac{\partial u}{\partial x}-u F\right)\right|_{(0, t)}=0 \text { for } t>0 \\
& \left.D\left(\frac{\partial u}{\partial x}-u F\right)\right|_{(1, t)}=0 \text { for } t>0 .
\end{aligned}
$$

We split the equation

$$
u_{t}=D\left(u_{x x}-u_{x} F-F_{x} u\right)
$$

into two parts as follows:

Diffusion part is

$$
u_{t}=D u_{x x}
$$

and advection-reaction part is

$$
u_{t}=-D u_{x} F-D F_{x} u \text {. }
$$

We then combine these equations by using the iterative splitting algorithm as follows:

$$
\begin{aligned}
u_{i} & =D\left(u_{i}\right)_{x x}-D\left(\left(u_{i-1}\right)_{x} F-F_{x} u_{i-1}\right), \\
u_{i+1} & =D\left(u_{i}\right)_{x x}-D\left(\left(u_{i+1}\right)_{x} F-F_{x} u_{i+1}\right),
\end{aligned}
$$

where $i=1,3, \ldots, 2 p+1$.

To solve these iterative schemes, we need initial and boundary conditions that are given as follows: For initial condition, we have

$$
u_{m}=1, \quad 0 \leqslant m \leqslant N,
$$


and for boundary conditions (13), (14), we have

$$
\begin{aligned}
D\left(\frac{\partial u_{0}}{\partial x}-u_{0} F_{0}\right) & =0 \text { for } t>0, \\
D\left(\frac{\partial u_{N}}{\partial x}-u_{N} F_{N}\right) & =0 \quad \text { for } t>0,
\end{aligned}
$$

where $m$ defines the spatial discretization step and $N$ is the spatial discretization number. The derivatives in Equations (21), (22) are approximated by using backward and forward difference formulas. The central difference approximation for each derivatives $u_{x x}$ and $u_{x}$ are taken into account for each grid point $\left(x_{m}, t\right)$ as follows:

$$
\left.\frac{\partial^{2} u}{\partial x^{2}}\right|_{\left(x_{m}, t\right)} \approx \frac{1}{h^{2}}\left(u_{m+1}(t)-2 u_{m}(t)+u_{m-1}(t)\right)
$$

and

$$
\left.\frac{\partial u}{\partial x}\right|_{\left(x_{m}, t\right)} \approx \frac{1}{2 h}\left(u_{m+1}(t)-u_{m-1}(t)\right),
$$

where $h$ is the spatial stepping and $m=0,1, \ldots, N$.

After assembling the unknowns of (23) and embedding the boundary conditions (21), (22), we have the following system of equations:

$$
u_{x x}=A_{1} u,
$$

where

$$
A_{1}=\frac{1}{h^{2}}\left(\begin{array}{ccccc}
-2+\left(1-h F_{0}\right) & 1 & 0 & 0 & 0 \\
1 & -2 & 1 & 0 & 0 \\
\vdots & \vdots & \ddots & \vdots & 0 \\
0 & 0 & 1 & -2 & 1 \\
0 & 0 & 0 & 1 & -2+\left(1+h F_{N}\right)
\end{array}\right)_{(N+1) \times(N+1)},
$$

and after assembling the unknowns of (24), we obtain the following system:

$$
u_{x}=B_{1} u,
$$

where

$$
B_{1}=\frac{1}{2 h}\left(\begin{array}{ccccc}
-\left(1-h F_{0}\right) & 1 & 0 & 0 & 0 \\
-1 & 0 & 1 & 0 & 0 \\
\vdots & \vdots & \ddots & \vdots & 0 \\
0 & 0 & -1 & 0 & 1 \\
0 & 0 & 0 & -1 & \left(1+h F_{N}\right)
\end{array}\right)_{(N+1) \times(N+1)}
$$

We fix the functions $F(x)$ and $F^{\prime}(x)$ at each discretization point $m=0,1, \ldots, N$ and have

$$
F(\underline{\mathrm{x}})=\left(\begin{array}{cccc}
F\left(x_{0}\right) & 0 & \ldots & 0 \\
0 & F\left(x_{1}\right) & \ldots & 0 \\
\vdots & \vdots & \ddots & \vdots \\
0 & \ldots & 0 & F\left(x_{N}\right)
\end{array}\right), \quad F^{\prime}(\underline{\mathrm{x}})=\left(\begin{array}{cccc}
F^{\prime}\left(x_{0}\right) & 0 & \ldots & 0 \\
0 & F^{\prime}\left(x_{1}\right) & \ldots & 0 \\
\vdots & \vdots & \ddots & \vdots \\
0 & \ldots & 0 & F^{\prime}\left(x_{N}\right)
\end{array}\right) .
$$

Note that we use central difference approximation for each $F^{\prime}\left(x_{m}\right)$. 
After redefining Equations (18), (19), we have linear bounded systems

$$
\begin{array}{r}
u_{i}^{\prime}=A u_{i}+B u_{i-1}, \\
u_{i+1}^{\prime}=A u_{i}+B u_{i+1},
\end{array}
$$

where $A=D A_{1}, B=-D F(\underline{\mathrm{x}}) B_{1}-D F^{\prime}(\underline{\mathrm{x}})$.

We then solve Equations (27), (28) by using the midpoint method on each subinterval $\left[t^{n}, t^{n+1}\right]$ where $n=0,1, \ldots, M$. Hence, the algorithms can be read as:

$$
\begin{aligned}
& u_{i}^{n+1}=\left(I-\frac{\tau}{2} A\right)^{-1}\left(\left(I+\frac{\tau}{2} A\right) u_{i}^{n}+\frac{\tau}{2} B\left(u_{i-1}^{n}+u_{i-1}^{n+1}\right)\right), \\
& u_{i+1}^{n+1}=\left(I-\frac{\tau}{2} B\right)^{-1}\left(\left(I+\frac{\tau}{2} B\right) u_{i+1}^{n}+\frac{\tau}{2} A\left(u_{i}^{n}+u_{i}^{n+1}\right)\right),
\end{aligned}
$$

where $\tau$ is the time discretzation step. We start iteration with $i=1$, initial guess $u_{0}(t)=0$ and initial conditions $u_{1}(t)=u_{0}$ and $u_{2}(t)=u_{0}$.

\section{ERROR ANALYSIS OF ITERATIVE SPLITTING METHOD TO MATHEMATICAL MODEL}

The local splitting error bounds are derived explicitly in the first theorem and we compare the errors of the iterative splitting method with the midpoint rule with respect to the midpoint rule without splitting in the second theorem. Here, $A$ and $B$ are linear bounded operators, where $A, B: X \rightarrow X$, $X$ is a Banach space considered with suitable matrix norm $\|\cdot\|$. (In this study, $X$ is equal to $R^{n \times n}$.)

\section{Theorem 5.1}

Let $A, B \in \mathscr{L}(X)$ be given linear bounded operators. The Cauchy problem is in (6). Then the problem has a unique solution. The error bounds of the iterations (8), (9) are given by

for $i$ odd

$$
\left\|\varepsilon_{i}\right\| \leqslant\left(K_{1} \cdot\|A\|\right)^{\frac{i-1}{2}} \cdot\left(K_{2} \cdot\|B\|\right)^{\frac{i+1}{2}} \cdot\left\|\varepsilon_{0}\right\| \frac{t^{i}}{i !}
$$

for $i$ even

$$
\left\|\varepsilon_{i}\right\| \leqslant\left(K_{1} \cdot\|A\|\right)^{\frac{i}{2}} \cdot\left(K_{2} \cdot\|B\|\right)^{\frac{i}{2}} \cdot\left\|\varepsilon_{0}\right\| \frac{t^{i}}{i !},
$$

where $\left\|\varepsilon_{0}\right\|$ is the difference between the exact solution and initial guess, $\|\exp (A t)\| \leqslant K_{1}$, $\|\exp (B t)\| \leqslant K_{2}$ for $t \geqslant 0$.

Proof

The algorithms of the method are given by

$$
\begin{aligned}
u_{i}^{\prime}(t) & =A u_{i}(t)+B u_{i-1}(t), \\
u_{i+1}^{\prime}(t) & =A u_{i}(t)+B u_{i+1}(t),
\end{aligned}
$$

with initial conditions $u_{i}(0)=u_{0}$ and $u_{i+1}(0)=u_{0}$ where $i=1,3, \ldots, 2 p+1$ for $[0, t]$.

For the first iteration, from the variation of constant formula, we have

$$
\Longrightarrow u_{1}(t)=\mathrm{e}^{A t} u_{0}+\int_{0}^{t} \mathrm{e}^{A(t-s)} B u_{0} \mathrm{~d} s,
$$

and we know the exact solution

$$
\Longrightarrow u(t)=\mathrm{e}^{A t} u_{0}+\int_{0}^{t} \mathrm{e}^{A(t-s)} B \mathrm{e}^{(A+B) s} u_{0} \mathrm{~d} s .
$$


For the second iteration, from the variation of constant formula, we have

$$
\Longrightarrow u_{2}(t)=\mathrm{e}^{B t} u_{0}+\int_{0}^{t} \mathrm{e}^{B(t-s)} A u_{1} \mathrm{~d} s
$$

and we know the exact solution

$$
\Longrightarrow u(t)=\mathrm{e}^{B t} u_{0}+\int_{0}^{t} \mathrm{e}^{B(t-s)} A e^{(A+B) s} u_{0} \mathrm{~d} s .
$$

Let us denote $\varepsilon_{i}=u(t)-u_{i}(t)$. Assume that $A, B$ are linear bounded operators and $\|\exp (A t)\| \leqslant K_{1},\|\exp (B t)\| \leqslant K_{2}$ for $t \geqslant 0$.

For $i=1$, we have the error bound

$$
\begin{aligned}
\left\|u(t)-u_{1}(t)\right\| & =\left\|\int_{0}^{t} \mathrm{e}^{A(t-s)} B\left(\mathrm{e}^{(A+B) s} u_{0}-u_{0}\right) \mathrm{d} s\right\|, \\
\left\|\varepsilon_{1}\right\| & =\left\|\int_{0}^{t} \mathrm{e}^{A(t-s)} B \varepsilon_{0} \mathrm{~d} s\right\|, \\
\left\|\varepsilon_{1}\right\| & \leqslant K_{1} .\|B\| \cdot\left\|\varepsilon_{0}\right\| t,
\end{aligned}
$$

for $i=2$, we get

$$
\begin{aligned}
\left\|u(t)-u_{2}(t)\right\| & =\left\|\int_{0}^{t} \mathrm{e}^{B(t-s)} A\left(\mathrm{e}^{(A+B) s} u_{0}-u_{1}\right) \mathrm{d} s\right\|, \\
\left\|\varepsilon_{2}\right\| & =\left\|\int_{0}^{t} \mathrm{e}^{B(t-s)} A\left(\varepsilon_{1}\right) \mathrm{d} s\right\|, \\
\left\|\varepsilon_{2}\right\| & \leqslant K_{2} \int_{0}^{t}\|A\| \cdot\left\|\varepsilon_{1}\right\| \mathrm{d} s, \\
\left\|\varepsilon_{2}\right\| & \leqslant K_{2} \cdot K_{1} \cdot\|A\| \cdot\|B\| \cdot\left\|\varepsilon_{0}\right\| \frac{t^{2}}{2}
\end{aligned}
$$

and for $i=3$

$$
\begin{aligned}
\left\|u(t)-u_{3}(t)\right\| & =\left\|\int_{0}^{t} \mathrm{e}^{A(t-s)} B\left(\mathrm{e}^{(A+B) s} u_{0}-u_{2}\right) \mathrm{d} s\right\|, \\
\left\|\varepsilon_{3}\right\| & =\left\|\int_{0}^{t} \mathrm{e}^{A(t-s)} B \varepsilon_{2} \mathrm{~d} s\right\|, \\
\left\|\varepsilon_{3}\right\| & \leqslant K_{1} \int_{0}^{t}\|B\| \cdot\left\|\varepsilon_{2}\right\| \mathrm{d} s, \\
\left\|\varepsilon_{3}\right\| & \leqslant K_{1} \cdot K_{2} \cdot K_{1} \cdot\|B\| \cdot\|A\| \cdot\|B\| \cdot\left\|\varepsilon_{0}\right\| \frac{t^{3}}{6},
\end{aligned}
$$

then by induction we get:

for $i$ odd

$$
\left\|\varepsilon_{i}\right\| \leqslant\left(K_{1} \cdot\|A\|\right)^{\frac{i-1}{2}} .\left(K_{2} .\|B\|\right)^{\frac{i+1}{2}} \cdot\left\|\varepsilon_{0}\right\| \frac{t^{i}}{i !}
$$

for $i$ even

$$
\left\|\varepsilon_{i}\right\| \leqslant\left(K_{1} \cdot\|A\|\right)^{\frac{i}{2}} \cdot\left(K_{2} \cdot\|B\|\right)^{\frac{i}{2}} \cdot\left\|\varepsilon_{0}\right\| \frac{t^{i}}{i !} .
$$

Note that in [5] they give the same error bounds implicitly, but here we write them in an explicit form. 
Theorem 5.2

The local error of the iterative splitting method based on the midpoint rule has more accuracy than the error of midpoint without splitting.

Proof

The error of iterative splitting with the midpoint rule is

$$
\left\|u_{\text {spl }}(t)-u_{\text {exact }}(t)\right\| \leqslant\|A B(A+B)\| \frac{t^{3}}{6}\left\|u_{0}\right\|
$$

and without splitting is

$$
\left\|u_{\text {mid }}(t)-u_{\text {exact }}(t)\right\| \leqslant\|A+B\|^{3} \frac{t^{3}}{12}\left\|u_{0}\right\| .
$$

Let

$$
\left\|\varepsilon_{\mathrm{spl}}\right\|=\left\|u_{\mathrm{spl}}(t)-u_{\mathrm{exact}}(t)\right\| \leqslant\|A\| \cdot\|B\| \cdot\|A+B\| \frac{t^{3}}{6}\left\|u_{0}\right\|
$$

and

$$
\left\|\varepsilon_{\text {mid }}\right\|=\left\|u_{\text {mid }}(t)-u_{\text {exact }}(t)\right\| \leqslant\|A+B\|^{3} \frac{t^{3}}{12}\left\|u_{0}\right\|,
$$

then we have estimation

$$
\frac{\left\|\varepsilon_{\text {spl }}\right\|}{\left\|\varepsilon_{\text {mid }}\right\|} \leqslant 1
$$

where $2\|A\| .\|B\| \leqslant\|A+B\|^{2}$.

\section{STABILITY ANALYSIS OF THE ITERATIVE SPLITTING METHOD TO MATHEMATICAL MODEL}

In this section, we explain the stability of the algorithms (29), (30) with the following theorem.

\section{Theorem 6.1}

The algorithms (29), (30) are stable with $Z_{1}=\tau A$ and $Z_{2}=\tau B$ if and only if there exists functions $R_{i}\left(Z_{1}, Z_{2}\right)$ such that:

$$
\begin{aligned}
& \left\|R_{i}\left(Z_{1}, Z_{2}\right)\right\| \leqslant 1+K \tau \\
& \quad \text { for all } Z_{1}, Z_{2} \in X \times X,
\end{aligned}
$$

where $K$ is a constant (independent of $\tau, h$ ), $X$ a Banach-space and $\|\cdot\|$ a matrix norm.

Proof

Discretizations of Equations (27) and (28) by the midpoint rule are given as

$$
\begin{aligned}
& u_{i}^{n+1}=\left(I-\frac{\tau}{2} A\right)^{-1}\left(I+\frac{\tau}{2} A\right) u_{i}^{n}+\left(I-\frac{\tau}{2} A\right)^{-1} \frac{\tau}{2} B\left(u_{i-1}^{n}+u_{i-1}^{n+1}\right), \\
& u_{i+1}^{n+1}=\left(I-\frac{\tau}{2} B\right)^{-1}\left(I+\frac{\tau}{2} B\right) u_{i+1}^{n}+\left(I-\frac{\tau}{2}\right)^{-1} \frac{\tau}{2} A\left(u_{i}^{n}+u_{i}^{n+1}\right) .
\end{aligned}
$$

We compute the first iteration with $i=1$ and get the equations

$$
\begin{aligned}
& u_{1}^{n+1}=R\left(Z_{1}\right) u_{1}^{n}+\tau\left(I-\frac{Z_{1}}{2}\right)^{-1} \frac{B}{2}\left(u_{0}^{n}+u_{0}^{n+1}\right), \\
& u_{2}^{n+1}=R\left(Z_{2}\right) u_{2}^{n}+\tau\left(I-\frac{Z_{2}}{2}\right)^{-1} \frac{A}{2}\left(u_{1}^{n}+u_{1}^{n+1}\right),
\end{aligned}
$$


where

$$
R(Z)=\left(I-\frac{Z}{2}\right)^{-1}\left(I+\frac{Z}{2}\right)
$$

and $Z_{1}=\tau A, Z_{2}=\tau B$.

\section{Remark 6.1}

The stability region for $R(Z)=(I-Z / 2)^{-1}(I+Z / 2)$ is precisely the left-half of the complex plane and $\|R(Z)\| \leqslant 1$ for this region. Proof is in [3].

After setting $u_{1}^{n}=u_{2}^{n}=u^{n}$ and initializing with $u_{0}^{n+1}=u_{0}^{n}=u^{n}$, then for the first part, we have the stability equation

$$
u_{1}^{n+1}=\left(R\left(Z_{1}\right)+\tau\left(I-\frac{Z_{1}}{2}\right)^{-1}\right) B u^{n} .
$$

With the prestep we obtain the stable function:

$$
\left\|\tilde{R}_{1}\left(Z_{1}, \tau\right)\right\| \leqslant 1+\tau K_{1},
$$

where $K_{1}=\left\|\left(I-Z_{1} / 2\right)^{-1}\right\| .\|B\|$ which is independent of $\tau$ and $h$.

After setting $u_{1}^{n}=u_{2}^{n}=u^{n}$ and putting $u_{1}^{n+1}=\tilde{R}_{1} u^{n}$, then for the second part, we have the stability equation

$$
u_{2}^{n+1}=\left(R\left(Z_{2}\right)+\tau\left(I-\frac{Z_{2}}{2}\right)^{-1} \frac{A}{2}+\tau\left(I-\frac{Z_{2}}{2}\right)^{-1} \frac{A}{2} \tilde{R}_{1}\left(Z_{1}, \tau\right)\right) u^{n} .
$$

With the prestep, we obtain the stable function:

$$
\left\|\tilde{R}_{2}\left(Z_{1}, Z_{2}, \tau\right)\right\| \leqslant 1+\tau\left\|\left(I-\frac{Z_{2}}{2}\right)^{-1}\right\| \cdot\|A\|+\frac{\tau^{2}}{2}\left\|\left(I-\frac{Z_{2}}{2}\right)^{-1}\right\| \cdot\|A\| \cdot K_{1} .
$$

Since $\tau^{2}<(T+1) \tau$ for $\tau \in[0, T]$, then

$$
\left\|\tilde{R}_{2}\left(Z_{1}, Z_{2}, \tau\right)\right\| \leqslant 1+\tau K_{2},
$$

where $K_{2}=\left\|\left(I-Z_{2} / 2\right)^{-1}\right\| .\|A\| .\left(1+K_{1}(T+1) / 2\right)$, which is independent of $\tau$ and $h$.

\section{Remark 6.2}

A one-step finite difference scheme (with constant coefficients) is stable in a stability region $\Lambda$ if and only if there is a constant $K$ (independent of $\theta, \tau$ and $h$ ) such that

$$
|g(\theta, \tau, h)| \leqslant 1+K \tau
$$

with $(\tau, h) \in \Lambda$. Proof is in [12].

\section{NUMERICAL RESULTS}

We present the application of the iterative operator splitting method for the numerical solution of a mathematical model for capillary formation in tumor angiogenesis. For numerical computation, we consider the problem (1)-(4) with parameters $D=0.00025, a=1, b=2, c=10, d=0.1, \alpha_{1}=\alpha_{2}=1$, $A_{1}=28 \times 10^{7}, A_{2}=0.22 \times 10^{9}$ and $k=16$. We write the computer program in Matlab and present our results on graphics that define the concentration of Endothelial Cells at different times.

In Figure 1, $u(x, t)$ is plotted for different values of $T$. It is seen that graphs, in Figure 1, show similar trends as the ones obtained by the method of lines in [10] and the tau method in [11]. In Tables I and II, we compare the errors of different splitting methods and the method of 

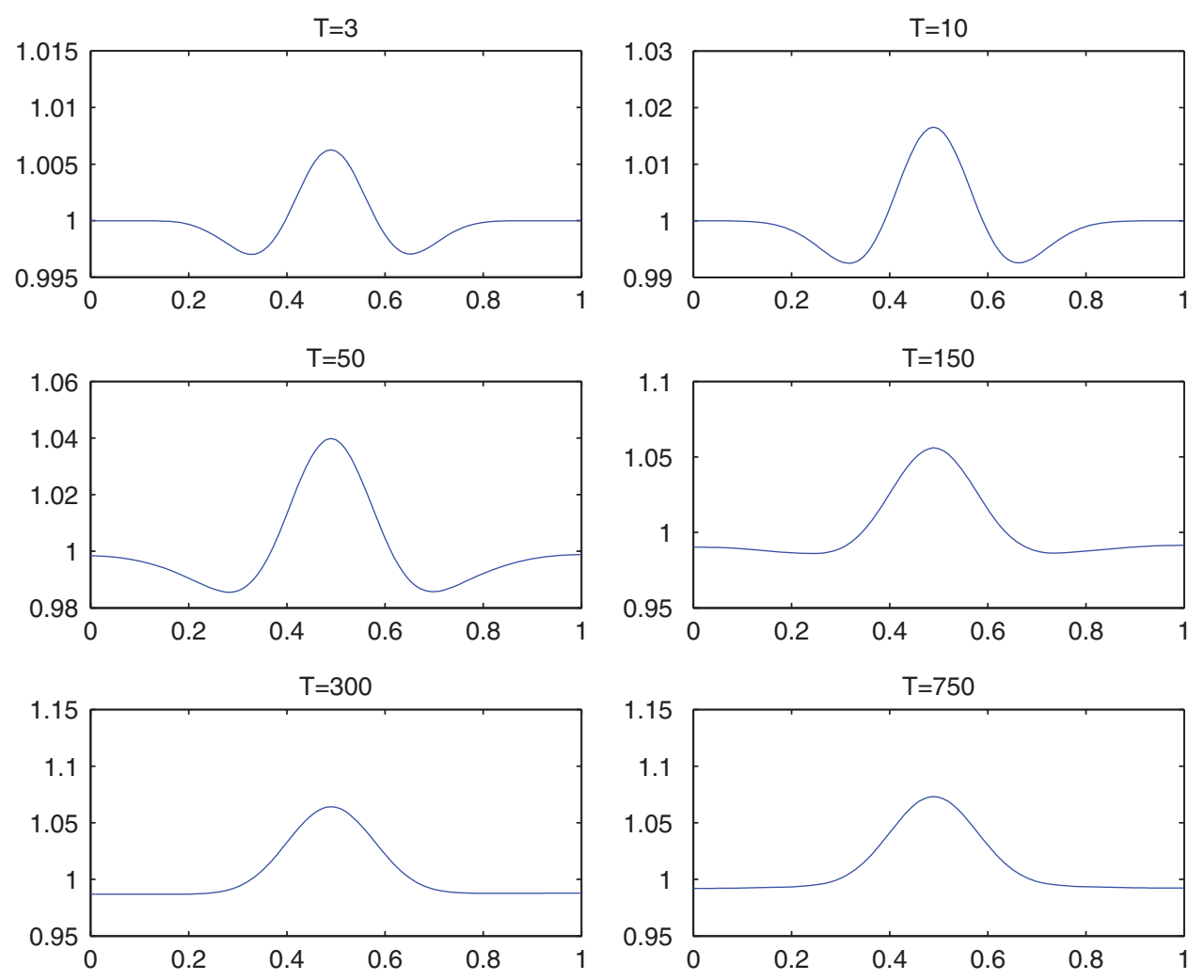

Figure 1. Numerical solution of the problem (1)-(4) by using iterative operator splitting methods for $T=3, T=10, T=50, T=150, T=300$ and $T=750$.

Table I. The errors of different splitting methods and the method of lines for $\Delta x=0.01, \Delta t=0.3$ at $T=150$.

\begin{tabular}{lccc}
\hline & $\operatorname{err}_{L_{1}}$ & $\operatorname{err}_{L_{2}}$ & $\operatorname{err}_{L_{\text {inf }}}$ \\
\hline Iterative splitting & $4.7701 \mathrm{e}-007$ & $5.3524 \mathrm{e}-008$ & $9.4143 \mathrm{e}-009$ \\
Sequential splitting & 0.0082 & 0.0013 & $3.5973 \mathrm{e}-004$ \\
Strang splitting & $2.8190 \mathrm{e}-005$ & $4.4547 \mathrm{e}-006$ & $1.1447 \mathrm{e}-006$ \\
Method of lines & $9.6361 \mathrm{e}-004$ & $1.0662 \mathrm{e}-004$ & $1.6967 \mathrm{e}-005$ \\
\hline
\end{tabular}

Table II. The errors of different splitting methods and the method of lines for $\Delta x=0.01, \Delta t=0.3$ at $T=300$.

\begin{tabular}{lccc}
\hline & $\operatorname{err}_{L_{1}}$ & $\operatorname{err}_{L_{2}}$ & $\operatorname{err}_{L_{\text {inf }}}$ \\
\hline Iterative splitting & $1.9941 \mathrm{e}-007$ & $2.2098 \mathrm{e}-008$ & $3.3364 \mathrm{e}-009$ \\
Sequential splitting & 0.0081 & 0.0013 & $3.7108 \mathrm{e}-004$ \\
Strang splitting & $2.9793 \mathrm{e}-005$ & $4.5777 \mathrm{e}-006$ & $1.0991 \mathrm{e}-006$ \\
Method of lines & $4.7212 \mathrm{e}-004$ & $5.2296 \mathrm{e}-005$ & $7.4543 \mathrm{e}-006$ \\
\hline
\end{tabular}

lines at times $T=150$ and $T=300$. It is shown that the iterative splitting method provides very accurate numerical solution for mathematical model in comparison with other classical splitting methods and the method of lines without splitting. In Figure 2, we simulate the solutions taken with different splitting methods at time $T=300$. In Tables III and IV, we compare the errors of iterative splitting and the difference method without splitting at times $T=3$ and $T=30$. We see that 


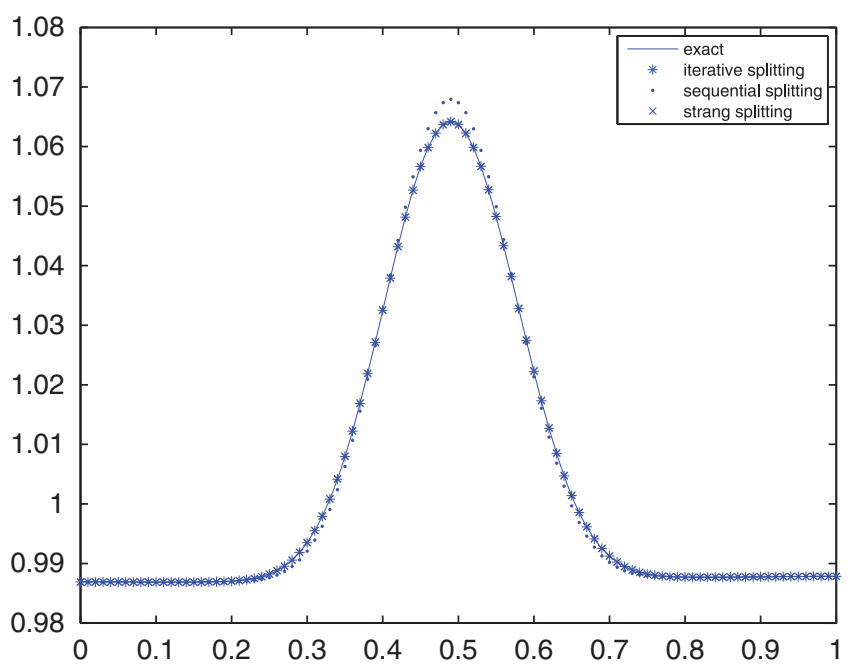

Figure 2. The comparison of solutions of the problem (1)-(4) for $\Delta x=0.01, \Delta t=0.3$ at $T=300$.

Table III. The errors of different splitting methods and the difference method (without splitting) for $\Delta x=0.01, \Delta t=0.03$ at $T=3$.

\begin{tabular}{lccc}
\hline & $\operatorname{err}_{L_{1}}$ & $\operatorname{err}_{L_{2}}$ & $\operatorname{err}_{L_{\infty}}$ \\
\hline Iterative splitting & $9.7647 \mathrm{e}-008$ & $1.4602 \mathrm{e}-008$ & $3.7775 \mathrm{e}-009$ \\
Difference method & $3.5514 \mathrm{e}-004$ & $6.4051 \mathrm{e}-005$ & $1.7128 \mathrm{e}-005$ \\
\hline
\end{tabular}

Table IV. The errors of different splitting methods and the difference method (without splitting) for $\Delta x=0.01, \Delta t=0.03$ at $T=30$.

\begin{tabular}{lccc}
\hline & $\operatorname{err}_{L_{1}}$ & $\operatorname{err}_{L_{2}}$ & $\operatorname{err}_{L_{\infty}}$ \\
\hline Iterative splitting & $6.6943 \mathrm{e}-008$ & $8.6847 \mathrm{e}-009$ & $1.9516 \mathrm{e}-009$ \\
Difference method & 0.0176 & 0.0027 & $5.5020 \mathrm{e}-004$ \\
\hline
\end{tabular}

iterative splitting gives better results than the difference method and also note that the difference method does not work at long times with big time-steps. Note that Matlab package expm is used as exact solution.

\section{CONCLUSION}

In this paper, we have presented the application of the iterative operator splitting method for the numerical solution of a mathematical model for capillary formation in tumor angiogenesis. We study the convergency properties of the method by using matrix analysis. We then compare the performance of the iterative splitting method with traditional operator splitting methods (sequential and Strang splitting) and non-splitting methods (method of lines and finite difference). The numerical results reveal that the iterative splitting method is applicable to this model problem and provides better accuracy compared to the other splitting methods and non-splitting methods.

The iterative splitting method is superior to the others, because of the following reasons:

- It includes all operators in each subequation unlike the traditional operator splitting methods. This is physically the best and hence we obtain the consistent approximations after each inner step. 
- It reduces the local splitting error by using more iteration steps to obtain higher order accuracy.

- It has a small constant in the local splitting error with respect to the method of lines.

- It gives a better performance at long time with respect to the finite difference method.

As a result, this application shows that the iterative operator splitting method gives high convergence and small error and it is quite easy to apply to the model problem. The consistency and stability analysis are also studied easily. In future work, we want to apply the method to higher dimensional problems.

\section{REFERENCES}

1. Geiser J. Decomposition Methods for Differential Equations: Theory and Application. CRC Press, Taylor \& Francis Group: Boca Raton, London, 2008.

2. Geiser J. Iterative operator-splitting methods with higher-order time integration methods and applications for parabolic partial differential equations. Journal of Computational and Applied Mathematics 2008; 217:227-242.

3. Hundsdorfer WH, Verwer JG. Numerical Solution of Time-dependent Advection-diffusion-reaction Equations. Springer: Berlin, 2003.

4. Faragó I, Geiser J. Iterative operator-splitting methods for linear problems. International Journal of Computational Science and Engineering 2007; 3:255-263.

5. Faragó I, Gnandt B, Havasi Á. Additive and iterative operator splitting methods and their numerical investigation. Computers and Mathematics with Applications 2008; 55:2266-2279.

6. Faragó I, Thomsen PG, Zlatev Z. On the additive splitting procesures and their computer realization. Applied Mathematical Modelling 2008; 32(8):1552-1569.

7. Levine HA, Pamuk S, Sleeman BD, Nilsen-Hamilton M. Mathematical model of capillary formation and development in tumor angiogenesis: penetration into the stroma. Bulletin of Mathematical Biology 2001; 63(5):801-863.

8. David D. Reinforced random walks. Probability Theory Related Fields 1990; 84:203-229.

9. Othmer HG, Stevens A. Aggregation, blow up and collapse: the ABC's oftaxis and reinforced random walks. SIAM Journal on Applied Mathematics 1997; 57(4):1044-1081.

10. Pamuk S, Erdem A. The method of lines for the numerical solution of a mathematical model for capillary formation: the role of endothelial cells in capillary. Applied Mathematics and Computation 2007; 186:831-835.

11. Saadatmandi A, Dehghan M. Numerical solution of mathematical model for capillary formation tumor angiogenesis via the tau method. Communications in Numerical Methods in Engineering 2008; 24:1467-1474.

12. Strikwerda JC. Finite Difference Schemes and Partial Differential Equations. SIAM: Philadelphia, PA, U.S.A., 2004. 